\title{
Prevalência de Síndrome de Burnout em médicos residentes de um hospital de ensino
}

\author{
Prevalence of Burnout syndrome in resident physicians of a teaching hospital \\ Prevalencia del síndrome de Burnout en médicos residentes de un hospital docente
}

Deborah Holanda da Silva Brayde ${ }^{1 *}$, Tanise Nazaré Maia Costa ${ }^{1}$.

\begin{abstract}
RESUMO
Objetivo: O objetivo deste estudo é identificar a prevalência de síndrome de burnout em médicos residentes do Hospital Ophir Loyola. Métodos: É um estudo quantitativo, descritivo e transversal. Utilizou-se um questionário de 30 questões, 8 com perguntas sobre o perfil sociodemográfico da população estudada e 22 questões retiradas da versão em português validada do Maslach Burnout Inventory (MBI). Utilizou-se o método de estatística descritiva para analisar os dados. A pesquisa foi submetida ao Comitê de Ética em Pesquisa e aprovada, pelo parecer 2.473.827. Resultados: Como resultados obteve-se um perfil formado por mulheres, na faixa-etária entre 25 e 29 anos, solteiras, procedentes de faculdades públicas e do estado do estado do Pará, tendo como fonte de renda a bolsa de residência e plantões externos, cursando o segundo ano de residência e raramente praticam atividades de lazer. Conclusão: A prevalência de síndrome de burnout foi de $6,4 \%$, com alto nível de exaustão emocional, baixo nível de realização profissional e alto nível de despersonalização.
\end{abstract}

Palavras-chave: Educação médica, Residência médica, Síndrome de burnout.

\begin{abstract}
Objective: This study is aimed to identify the prevalence of burnout syndrome in medical residents from Ophir Loyola Hospital. Methods: It is a quantitative, descriptive and cross-sectional study. A questionnaire composed by 30 questions was used, 8 with questions about the socio-demographic profile focused on the studied population and 22 questions taken from the validated Portuguese version of the Maslach Burnout Inventory (MBI). The descriptive method was used to analyze the statistics about data. The research was submitted to the Ethics Committee in Research and approved, by the opinion 2,473,827. Results: As a result, a profile was obtained by women, ranging from 25 to 29 years of age, who were single, coming from public schools and from the state of Pará, having as their source of income the residence scholarship and external shifts, attending the second year of residence and rarely practice leisure activities. Conclusion: The prevalence of burnout syndrome was $6.4 \%$, with a high level of emotional exhaustion, low level of professional achievement and high level of depersonalization.
\end{abstract}

Key words: Medical education, Medical residence, Burnout syndrome.

\section{RESUMEN}

Objetivo: El objetivo de este estudio es identificar la prevalencia del síndrome de Burnout en médicos residentes del hospital Ophir Loyola. Métodos: Se trata de un estudio cuantitativo, descriptivo y transversal. Se utilizó un cuestionario de 30 preguntas, 8 con preguntas sobre el perfil sociodemográfico de la población estudiada y 22 preguntas tomadas de la versión portuguesa validada del inventario de Burnout de Maslach

${ }^{1}$ Hospital Ophir Loyola. Belém - Pará. *E-mail: deborah brayde@yahoo.com.br

SUBMETIDO EM: 4/2019

ACEITO EM: 5/2019

PUBLICADO EM: 7/2019

REAS/EJCH | Vol. Sup.24 | e758 | DOI: https://doi.org/10.25248/reas.e758.2019 Página 1 de 7 
(MBI). El método estadístico descriptivo se utilizó para analizar los datos. La investigación fue presentada al Comité de ética de investigación y aprobada por el dictamen 2.473.827. Resultados: Como resultado, se obtuvo un perfil formado por mujeres, en el rango de edad entre 25 y 29 años, soltero, de colegios públicos y del estado de Pará, teniendo como fuente de ingresos la beca de residencia y turnos externos, atendiendo a la Segundo año de residencia y raramente practica actividades de ocio. Conclusión: La prevalencia del síndrome de Burnout fue de $6,4 \%$, con alto nivel de agotamiento emocional, bajo nivel de logro profesional y alto nivel de despersonalización.

Palabras clave: Educación médica, Residencia médico, Síndrome de burnout.

\section{INTRODUÇÃO}

A residência médica é um tipo de pós-graduação em forma de especialização considerada padrão-ouro na formação médica, devendo ser desenvolvida em instituições universitárias ou não, sob orientação de profissional médico com alto nível de qualificação ética e profissional. Constitui-se na prática de treinamento em serviço, com 1800 horas de atividades, sendo 4 horas semanais destinadas a atividades de ensino (BRASIL, 1977; DIAS BA, et al., 2016).

A residência médica é considerada a melhor alternativa de aperfeiçoamento e especialização na área da saúde, porém traz consigo muitos problemas de ordem física e emocional, podendo-se destacar: isolamento social, fadiga, depressão, estafa, sonolência, estresse; que podem repercutir na qualidade de vida e saúde desses profissionais, como na qualidade do serviço prestado, levando a caracterização da Síndrome de Burnout (SB) (BORMIO MF, 2016; PURIM KSM, et al., 2016).

A SB é caracterizada por sintomas físicos (fadiga, distúrbios do sono, dores musculares, queixas gastrointestinais e disfunções sexuais), psíquicos (falta de atenção, alterações de memória, desânimo, depressão), comportamentais (irritabilidade, agressividade, perda de iniciativa) e defensivos (tendência ao isolamento, sentimento de impotência, perda de interesse no trabalho, cinismo) (FONTE CMS, 2011).

A SB é considerada um sério problema de saúde do trabalhador, em que estudos internacionais demonstram uma prevalência de $50 \%$ a $70 \%$ entre residentes médicos no geral e $63 \%$ em residentes de clínica médica. No Brasil, observou-se sua existência em 78,4\% em residentes de ortopedia, clínica médica, cirurgia, pediatria, ginecologia e obstetrícia, em um hospital público pesquisado (FABICHAK C, 2014).

Destacam-se alguns fatores que podem se relacionar a esta síndrome, como: a sobrecarga de trabalho, tanto em termos quantitativos como qualitativos; relação entre os profissionais e os clientes; e relacionamento entre os colegas de trabalho e as chefias (FAGUNDES PS, 2016).

Diante da necessidade de submissão do profissional médico aos programas de residência e suas repercussões na qualidade de vida desses indivíduos, nos processos de trabalho e nos serviços de saúde prestados à população, faz-se de grande relevância estudar a sua prevalência, a fim de promover práticas de prevenção e amenização da SB.

Assim, a partir das experiências vividas como médica residente do programa de clínica médica de um hospital de referência em Belém, surgiu o interesse de conhecer o grau de prevalência de síndrome de burnout entre os residentes deste hospital de ensino no ano de 2017, distribuídos nos 10 programas oferecidos por esta instituição.

\section{MÉTODOS}

Esta pesquisa caracterizou-se como um estudo do tipo quantitativo, descritivo e transversal, realizada no Hospital Ophir Loyola, localizado na em Belém - Pará. Instituição que presta assistência médica em oncologia e outras 17 especialidades, desenvolve ainda atividades de ensino, pesquisa e extensão, qualificando profissionais do Estado do Pará. A população do estudo foi formada por médicos-residentes matriculados na 
divisão de ensino no ano de 2017, totalizando 94 profissionais, distribuídos nos seguintes programas: anestesiologia, clínica médica, cirurgia geral, cancerologia clínica, cancerologia cirúrgica, endoscopia, mastologia, hematologia e hemoterapia, urologia e radiologia.

Considerando-se um erro amostral de 5\%, estimou-se uma amostra significativa no total de pelo menos 76 residentes a serem submetidos à pesquisa, porém apenas 47 responderam o questionário.

Os critérios de inclusão estavam relacionados a estarem desenvolvendo regularmente suas atividades na residência no ano de 2017 e aceitarem participar da pesquisa.

Como critérios de exclusão, estavam relacionados à não execução regular das atividades no ano de 2017 (licenças ou desistências) e participação direta na pesquisa, excluindo-se assim 3 residentes do processo.

Como orienta a resolução 466 de 12 de dezembro de 2012, que trata da ética em pesquisa com seres humanos, foi submetido à análise pelo comitê de ética em pesquisa (CEP) do Hospital Ophir Loyola aprovado pelo parecer.

A participação da pesquisa ocorreu voluntariamente, através do aceite de um Termo de Consentimento Livre e Esclarecida (TCLE), onde estão dispostos os esclarecimentos e as informações necessários sobre a pesquisa.

Utilizou-se como instrumento de coleta de dados, um questionário com 30 questões, sendo as 8 primeiras com o objetivo de caracterizar o perfil sociodemográfico da população estudada e as 22 questões seguintes retiradas da versão em português validada do Maslach Burnout Inventory (MBI), sobre exaustão profissional, realização profissional e despersonalização, como explicitado por Fonte CMS (2011).

As respostas estão organizadas em escala tipo likert com 5 opções cada pergunta. Cada opção da escala recebe uma pontuação progressiva: nunca (1) e sempre (5), cuja a somatória terá a seguinte interpretação: 0 - 20 nenhum indício de burnout; 21-40 possibilidade de desenvolver burnout; 41-60 fase inicial de burnout; 61-80 burnout começa a se instalar; 81 - 100, fase considerável de burnout.

Os residentes foram convidados a participar voluntariamente da pesquisa através de mensagens de texto via celular e os questionários enviados para o e-mail, podendo ser respondidos on-line, utilizando-se o aplicativo Google Forms.

As respostas foram enviadas diretamente para o e-mail do pesquisador, mantendo a confidencialidade das respostas e sendo armazenadas para posterior análise. As informações da caracterização amostral foram apuradas em banco de dados elaborado no software Microsoff ${ }^{\circledR}$ Office Exce ${ }^{\circledR} 2010$.

A estatística analítica foi utilizada para avaliar os resultados das variáveis categóricas da amostra através do Teste G e Qui-Quadrado Aderência para tabelas univariadas e Testes G, Qui-Quadrado Independência e Partição para tabelas bivariadas. As estatísticas descritiva e analítica, foram realizadas no software BioEstat ${ }^{\circledR}$ 5.0 (AYRES et al., 2007).

Para a tomada de decisão, adotou-se o nível de significância $\alpha=0,05$ ou $5 \%$, sinalizando com asterisco $\left({ }^{*}\right)$ os valores significantes.

\section{RESULTADOS}

Do total de 47 residentes que optaram por participar da pesquisa, observou-se predominância do sexo feminino, com $57,4 \%$ na sua totalidade, seguido do sexo masculino $42,6 \%$. Distribuídos nas seguintes faixasetárias: 20 - 24 anos 6,4\%; 25 - 29 anos 59,6\%, 30 a 34 anos $31,9 \%$ e acima de 35 anos 2,1\%.

Em relação ao estado civil houve predominância entre os solteiros, na proporção de $57,4 \%$, seguido dos casados com $29,8 \%$, união estável $10,64 \%$ e divorciados $2,13 \%$. A maioria dos residentes são provenientes do estado do Pará, constituindo-se em um percentual de $63,8 \%$, seguido de $36,2 \%$ de outros estados e possuíam como fonte de renda: bolsa + plantões externos $70,2 \%$, bolsa + outras fontes $23,4 \%$ e apenas bolsa de residência $6,4 \%$ (Tabela 1 ). 
Tabela 1 - Perfil Sociodemográfico.

\begin{tabular}{|c|c|c|c|c|}
\hline & Gênero & Fonte de Renda & Faixa-etária & Estado Civil \\
\hline Feminino & $57,4 \%$ & & & \\
\hline Masculino & $42,6 \%$ & & & \\
\hline Solteiro & & & & $57,4 \%$ \\
\hline Casado & & & & $29,8 \%$ \\
\hline União estável & & & & 10,64 \\
\hline Divorciado & & & & 2,13 \\
\hline 20- 24 anos & & & $6,4 \%$ & \\
\hline 25-29 anos & & & $59,6 \%$ & \\
\hline 30-35 anos & & & $31,9 \%$ & \\
\hline$>35$ anos & & & $2,1 \%$ & \\
\hline Bolsa & & $6,4 \%$ & & \\
\hline Bolsa + outras fontes & & $23,4 \%$ & & \\
\hline Bolsa + Plantões & & $70,2 \%$ & & \\
\hline
\end{tabular}

Fonte: Protocolos de pesquisa, 2018.

No variável ano de residência, a grande maioria (34\%) estava no primeiro ano de residência, seguido do segundo ano $(27,7 \%$ ), terceiro ano com $25,5 \%$, quarto ano $10,6 \%$ e o quinto ano $2,1 \%$. Com, $59,6 \%$ raramente praticavam atividades de lazer, $4,3 \%$ nunca as praticavam e $36,2 \%$ frequentemente as praticavam.

$\mathrm{Na}$ análise da síndrome de Burnout entre os pesquisados obteve-se a distribuição demonstrada no gráfico 3. Onde $80,9 \%$ apresentam a síndrome começando a se instalar, $12,8 \%$ estão na fase inicial de Burnout e $6,4 \%$ encontram-se em uma fase considerável de Burnout (Gráfico 1).

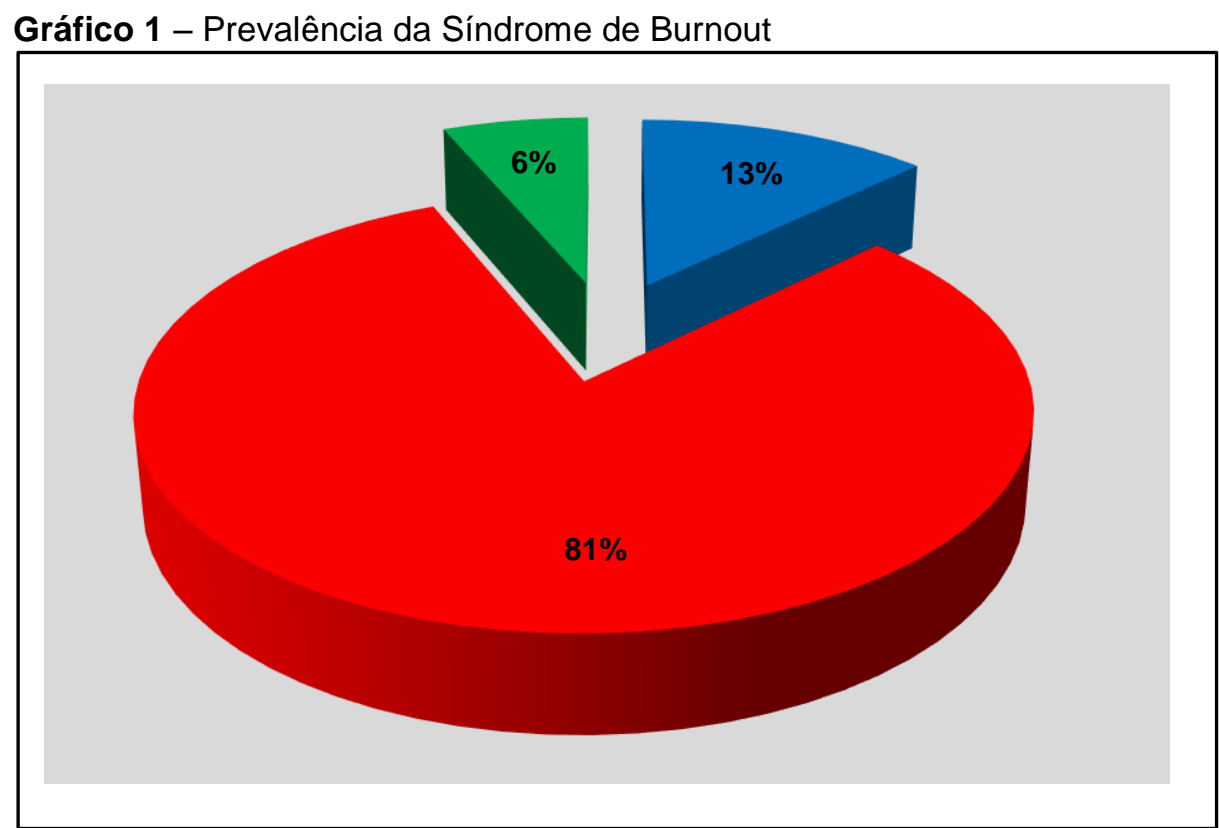

Fonte: Protocolos de pesquisa, 2018.

Legenda: Verde: Fase considerável de Burnourt 6\%, Azul: Fase inicial de Burnout $13 \%$, Vermelho: Burnout começa a se instalar $81 \%$. 
A relação entre a as fases da síndrome e o ano de residência, demonstrou maior prevalência quando o Burnout começa se instalar em todos os anos de residência, mas se instala principalmente entre os residentes do segundo ano. A fase inicial foi mais relevante entre os residentes do quarto ano. Não havendo diferença significativa na classificação de Burnout de acordo com ano de residência, podendo ser encontrado nas diversas fases do estágio.

Quanto as dimensões da síndrome, houve altos níveis de exaustão emocional e despersonalização entre os residentes, estando a realização profissional com os níveis mais baixos avaliados.

Gráfico 2 - Níveis de instalação e dimensões da Síndrome de Burnout.

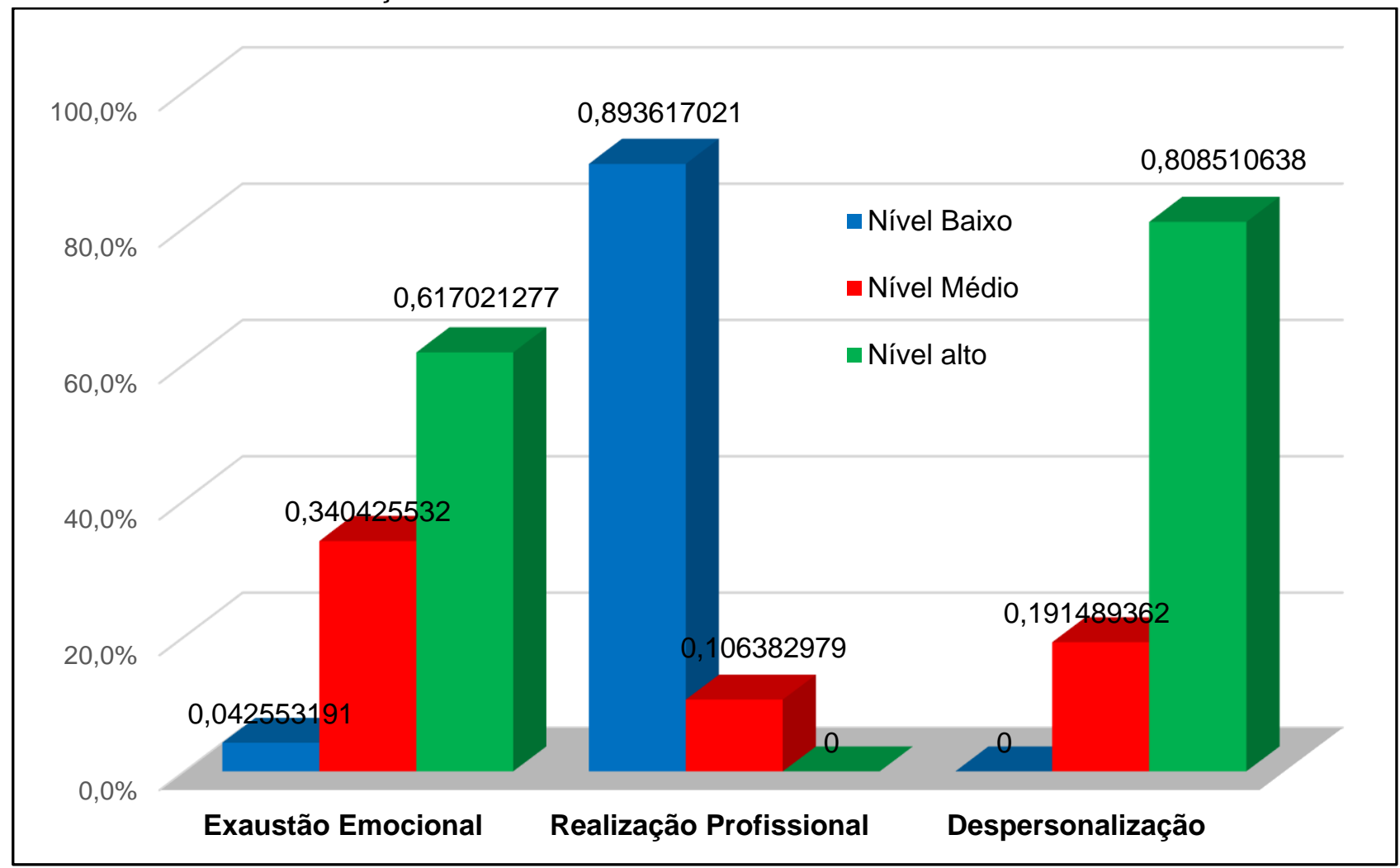

Fonte: Protocolos de pesquisa, 2018.

\section{DISCUSSÃO}

A síndrome de burnout é uma doença ocupacional que atinge profissionais de saúde e considerando sua importância no meio médico, objetiva-se identificar sua prevalência entre residentes de um hospital de ensino em Belém, comparando os resultados obtidos com a literatura disponível sobre o tema.

Por isso, considerando-se a distribuição de gêneros e comparando com a literatura analisada, houve discordância dos estudos de Asaiag PD, et al. (2009), Guido LA, et al. (2012), Fabichak C, et al. (2014) e Tavares KFA, et al. (2014), onde houve predominância de residentes do sexo feminino. Já em relação a faixa etária, assemelhou-se aos estudos de Asaiag PD, et al. (2009), Guido LA, et al. (2012) Fabichak C, et al. (2014) e Tavares KFA, et al. (2014), que tiveram a média de idade entre 26 e 31 anos.

Os resultados relacionados ao estado civil corroboraram com os estudos de Asaiag PD, et al. (2009), Guido LA, et al. (2012), Fabichak C, et al. (2014) e Tavares KFA, et al. (2014), em que também houve predominância de solteiros entre os residentes. A maioria dos residentes também são provenientes do estado do Pará, assim como relatado por Tavares KFA, et al. (2014), cujos residentes eram na grande maioria $(52,33 \%)$ procedentes do estado do Rio de Janeiro, ou seja, na própria cidade aonde realizavam a residência, reduzindo os custos com moradia e alimentação. 
Os residentes tiravam plantões além dos da residência, cerca de $70,2 \%$ possuíam essa prática. Não sendo estimada a carga-horária média semanal de trabalho dos residentes, mas considerando-se a carga-horária mínima da residência de 60 horas semanais, incluindo um máximo de 24 horas de plantão, como citado por Dias BA, et al. (2016) e a maioria realizando plantões externos, observa-se uma sobrecarga de atividades laborais, aumentando o risco de síndrome de burnout entre os profissionais.

Purim KSM, et al. (2016), também relatou que $89 \%$ dos residentes pesquisados por ela, realizavam uma média de 2 plantões semanais, 99\% possuíam outros vínculos de trabalho, totalizando uma média de 77 horas semanais de atividade profissional. E Dias BA, et al. (2016) observou que $66,7 \%$ possuíam outro emprego além da residência, que estaria relacionado ao baixo valor da bolsa e as excessivas despesas com moradia. Assim, para Purim KSM et al. (2016) e Dias BA, et al. (2016) a carga-horária extenuante de trabalho, totalizando as horas da residência e dos plantões externos, aumenta o risco de síndrome de burnout entre os residentes, pelo desgaste físico e emocional.

A distribuição entre os anos de residência foi semelhante ao encontrado por Fabichak C, et al. (2014), tendo como resultados a maioria residentes do primeiro ano. Já Asaiag PD, et al. (2010) e Dias BA, et al. (2015) a maioria dos pesquisados estavam no segundo ano de residência, demonstrando os altos níveis da síndrome nos primeiros anos de curso e sintomas de exaustão emocional mais comuns entre residentes do primeiro ano e diminuindo no decorrer dos anos.

A maioria dos residentes raramente possuem atividades de lazer, que quando comparado a Lourenção LG, et al. (2010), este relaciona a ausência de tempo para atividades de lazer como um dos fatores desencadeantes para a síndrome de burnout, aumentando o risco de desenvolvê-la entre os pesquisados. As longas jornadas de trabalho e pouco tempo para lazer e descanso foram relatados como causa de esgotamento profissional. A fadiga, sonolência, depressão, esgotamento, insatisfação no trabalho e baixa qualidade de vida, podem afetar negativamente a assistência, segurança e satisfação do paciente.

No estudo de Guido LA, et al. (2012), 20,8\% dos participantes residentes médicos apresentavam indicativo para síndrome de burnout. Desses, 65\% apresentavam alta exaustão emocional; $61,7 \%$ alta despersonalização e $30 \%$ baixa realização profissional. Mendoza-González et al (2016), obteve como resultados prevalência de $18,4 \%$ de Burnout, sendo 5,1\% de cansaço emocional, $11,2 \%$ despersonalização e $7,1 \%$ de baixa realização pessoal.

Asaiag PD, et al. (2010) observou alto nível de exaustão emocional e despersonalização e moderado nível de realização pessoal. Já para Fabichak C, et al. (2014) metade dos participantes apresentaram critérios para síndrome de burnout, com $75 \%$ com exaustão emocional e despersonalização e níveis baixos de realização profissional em $70,8 \%$.

Assim observa-se que os resultados encontrados, da prevalência de síndrome de burnout entre os médicos do Hospital Ophir Loyola, foram mais baixos que os relatados pela literatura, porém, a maioria não aderiu a pesquisa e evidenciou-se índice elevado da síndrome começando a se instalar naqueles entrevistados. A relação entre a as fases da síndrome e o ano de residência, demonstrou maior prevalência quando o Burnout começa se instalar em todos os anos de residência. Sendo a fase considerável maior entre os residentes do segundo ano, já a fase inicial foi mais relevante entre os residentes do quarto ano. Não havendo diferença significativa na classificação de acordo com ano de residência. Nas dimensões da síndrome de burnout desenvolvem-se primeiro a exaustão emocional, depois surge a despersonalização na tentativa de enfrentar a exaustão, seguido da redução da capacidade de resistir as demandas de trabalho e por fim, reduzindo os sentimentos de realização pessoal (GRACINO ME, et al., 2016).

Nesse estudo para as dimensões da análise da síndrome de Burnout, houve incidência significante de nível alto na dimensão da realização profissional, e houve incidência significante de nível alto nas dimensões da exaustão emocional e despersonalização. Corroborando com o estudo de Asaiag PD, et al. (2010), em que os residentes apresentaram alto nível de exaustação emocional e moderado nível de realização pessoal. Os quais podem ter sua sintomatologia amenizada por apoio das chefias, nas situações de sobrecarga do serviço e acompanhamento psicológico pelos serviços de residência. 


\section{CONCLUSÃO}

A partir das análises dos dados, chegou-se à conclusão que o perfil dos residentes do Hospital Ophir Loyola (HOL), é caracterizado por maioria do sexo feminino, na faixa-etária entre 25 e 29 anos, solteiros, procedentes de faculdades públicas e do estado do Pará, tendo como fonte de renda a bolsa de residência e plantões externos, cursando o segundo ano de residência e raramente praticam atividades de lazer. A prevalência da síndrome de burnout foi de 6,4\%, com 80,9\% apresentam a síndrome começando a se instalar. Os médicos tiveram alto nível de exaustão emocional, baixo nível de realização profissional e alto nível de despersonalização. Assim, síndrome de burnout está diretamente relacionada ao elevado estresse físico e psicológico do processo de formação da residência, que muitas vezes não pode ser evitado, como cargahorária elevada e desgaste emocional.

\section{REFERÊNCIAS}

1. AYRES M, et al. Bioestat 5.0 aplicações estatísticas nas áreas das ciências biológicas e médicas. Belém: IDSM, 2007; 364p.

2. ASAIAG PD, et al. Avaliação da qualidade de vida, sonolência diurna e burnout em médicos residentes. Revista Brasileira de Educação Médica, 2010; 3(34): 422-429.

3. BRASIL. CASA CIVIL. Decreto ํㅜ 80.281, de 5 de setembro de 1977. Diário Oficial, Brasília, DF, 5 de setembro. 1977.

4. BORMIO MF. Médicos residentes: estudo ergonômico a respeito da qualidade de vida e bem-estar. Pós-Doutorado (Relatório do programa de pós-doutorado em Qualidade de Vida) - Universidade Fernando Pessoa, Porto, 2016; $40 \mathrm{p}$.

5. DIAS BA, et al. Qualidade de vida de médicos residentes de um hospital escola. Scientia Medica, 2016; 1(26): 1 9.

6. FABICHAK C, et al. Síndrome de burnout em médicos residentes e preditores organizacionais do trabalho. Revista Brasileira de Medicina do Trabalho, 2014; 2(12): 79-84.

7. FAGUNDES PS. Síndrome de Burnout entre profissionais de saúde: uma revisão de literatura. Trabalho de Conclusão de curso (Especialização em Saúde do Trabalhador) - Universidade de Santa Cruz do Sul (UNISC), Santa Cruz do Sul. 2016; 20 p.

8. FONTE CMS. Adaptação e validação para português do questionário de Cppenhagen Burnout Inventory (CBI). Dissertação (Mestrado em Gestão e Economia de Saúde) - Universidade de Coimbra, Coimbra, 2011; 138 p.

9. GRACINO ME, et al. A saúde física e mental do profissional médico: uma revisão sistemática. Saúde debate, 2016; 40(140): 244-263.

10. GUIDO LA, et al. Síndrome de Burnout em residentes multiprofissionais de uma universidade pública. Revista da Escola de Enfermagem (USP), 2012, 6(46): 1477-1483.

11. LOURENÇÃO LG, et al. Saúde e qualidade de vida de médicos residentes. Revista da Associação Medica Brasileira, 2010; 1(56): 81-91.

12. MASLACH C, JACKSON SE. The measurement of experienced burnout. Journal of Occupacional Behavior, 1981; 2: $99-113$.

13. MENDOZA-GONZALEZ, et al. Desgaste professional, calidade de vida y salud en residentes de medicina, en el estado de Veracruz, México. Revista eletrônica Medicina, salud y sociedade, 2016; 6(2).

14. PURIM KSM, et al. Privação de sono e sonolência excessiva e médicos residentes e estudantes de medicina. Revista do Colégio Brasileiro de Cirurgiões, 2016, 6(43): 438-444.

15. TAVARES KFA, et al. Ocorrência de síndrome de burnout em enfermeiros residentes. Acta Paulista de Enfermagem, 2014; 3(27): 260-265. 